\title{
Phenotypic characterisation of immune cells associated with histological regression in cutaneous melanoma
}

\author{
Simona Osella-Abate ${ }^{1, *}$, Luca Conti ${ }^{1, *}$, Laura Annaratone ${ }^{1}$, \\ Rebecca Senetta ${ }^{1}$, Luca Bertero ${ }^{1}$, Matteo Licciardello ${ }^{2}$, \\ Virginia Caliendo ${ }^{3}$, Franco Picciotto ${ }^{3}$, Pietro Quaglino ${ }^{2}$, Paola Cassoni ${ }^{1}$, \\ SIMONE RIBERO ${ }^{2,3}$ \\ ${ }^{1}$ Department of Medical Sciences, Section of Surgical Pathology, University of Turin, Torino, \\ Italy; ${ }^{2}$ Department of Medical Sciences, Section of Dermatology, University of Turin, Torino, \\ Italy; ${ }^{3}$ Dermatologic Surgery Section, Department of Oncology, AOU Città della Salute e \\ della Scienza di Torino, Torino, Italy; * these authors equally contributed to the study
}

\begin{abstract}
Summary
Histological regression and tumour infiltrating lymphocytes represent an early sign of activation of the immune system against primary melanoma. The first phenomenon has been especially discussed in the literature because of its prognostic role, but no clear agreement on its evaluation has been reached. Immunotherapy of advanced stage melanoma has recently shown promising results; an improved understanding of the initial interplay between melanoma cells and the immune system would potentially help tailor treatment for patients.

Seventy consecutive melanomas with regression were analysed to identify a prognostic cut-off value of regression extension. Then, we compared the immune infiltrate between regressed and not regressed areas of these regressed melanomas, assessing CD3, CD4, CD8, CD20, CD123, PD1 and FOXP3/CD25 expression. The immune infiltrate of these cases was further compared with 28 control melanomas without regression.

A regression extension of $10 \%$ represented a reliable cutoff to distinguish two different risk categories in regressed melanomas. Regressed areas were less infiltrated by CD4/ CD25, FOXP3/CD4 or PD1/CD4 compared to not regressed areas of each sample. These lymphocyte subsets are associated with anergy and hamper the immune CD8+ response towards the cancer cells. Moreover, the relevance of these findings was further supported by the observation that not regressed controls were significantly more infiltrated by these anergic immune cell subsets compared to the regressed cases.

These results help understand the real meaning of regression in melanoma. Moreover, the association here identified between specific immunomodulatory immune cell subsets and regression could help in developing new therapeutic strategies.
\end{abstract}

Key words: Tumour infiltrating lymphocytes; melanoma; histological regression; survival.

Received 7 January, revised 18 March, accepted 31 March 2019 Available online 29 June 2019

\section{INTRODUCTION}

Despite extensive efforts aimed at identifying prognostic biomarkers in melanoma, the clinical behaviour of this tumour remains often unpredictable with frequent unexpected progressions, both in terms of timing and type. Additional markers are warranted to identify early stage patients at higher risk of recurrence, in order to offer patienttailored follow up or adjuvant treatments. Currently, evidence has shown that the specific malignancy and biological features of a cancer depend not only on its genetic abnormalities, but also on the interplay between cancer cells and microenvironment. The relationship between the tumour and host's immune system is particularly intriguing, especially considering that drugs targeting these pathways have shown significant clinical efficacy in multiple tumour types. ${ }^{2}$ Histological regression in melanoma is a paradigmatic example of this phenomenon. Some authors found no association between regression and melanoma prognosis, ${ }^{3}$ while other research groups reported a significant correlation between them. ${ }^{4-6}$ This discordance could be due to several factors including the heterogeneity of patient populations, as well as the different research methods applied, but what matters most is probably the lack of standardised histological criteria for its assessment. In particular, there is no agreement among pathologists in defining regression or in its sub-classification.

Tumour-infiltrating lymphocytes (TILs) have been identified as a favourable prognostic marker across multiple cancers including breast, ovarian, and colon cancer neoplasms and may serve as a surrogate indicator of the strength of the host anti-tumour immune response. ${ }^{8}$ TIL and histological regression are two distinct, sometimes overlapping, phenomena of the melanoma biology that still deserve better explanation in order to differentiate them.

The composition of the regression infiltrate has been partially characterised. Effector and suppressor T cells, B cells, natural killer cells, macrophages, and dendritic suppressor cells, can all impart competing immuno-stimulatory or immunosuppressive effects within the tumour microenvironment.

The first aim of this study was thus to describe and histologically characterise the regression phenomenon in melanoma, assessing its impact on survival. The second aim was 
to evaluate the phenotype of the infiltrating lymphocytes comparing regressed and not regressed areas in order to identify cell subsets related to the phenomenon of histological regression.

\section{METHODS}

\section{Study population}

A cohort of 70 consecutive patients with primary cutaneous melanoma attending the Dermatologic Clinic of the University of Turin between January 2003 and December 2014 were enrolled in the study. All patients signed a consent form to give permission to include their biological sample in a biobank, and the study was approved by the local ethics committee. In order to perform an optimal evaluation of the histomorphological parameters, only melanoma samples with complete removal of the lesion and in which the entire thickness of the neoplasm was submitted to histological examination were included. Therefore, shave and punch biopsies, partial excisions and samples with too little/poor material were excluded from the study. In addition, a second cohort of 28 not regressed melanomas was used to compare the immunophenotypic composition of the TIL. Patient demographics, clinical, pathological, and follow-up information were prospectively recorded and updated on an internal database. For each case, the inflammatory regression areas were assessed (TIL inside regression area, TIL-IRA). In addition, one or more areas including inflammatory cells intimately associated with the tumour cells outside the regression area (TIL-ORA) were analysed for each case if present, and in the control series (TIL-CTRL).

For full details regarding histopathological review, and immunophenotypical evaluation of TIL, ${ }^{2,9-13}$ see the Supplementary Methods (Appendix A).

\section{Morphological evaluation of histological regression ${ }^{13,14}$}

\section{Histological regression: definition and phases}

Regression was defined and classified as follows: (i) early phase as a derma tumour area with a dense mononuclear infiltrate composed mainly of lymphocytes, histiocytes and melanophages that actively infiltrate the neoplastic nests and at least partially replace neoplastic melanocytes; (ii) late phase as a dermal or epidermal area with disappearance of neoplastic cells and their replacement with dense fibrous tissue, melanophages, dilated vessels and focal or missing residual lymphocytic infiltration in the dermis.

\section{Histological regression: ratio/percentage}

Once the presence of one or more regression areas was established, the regression/tumour area ratio was calculated as the ratio between the linear extension of regression areas and tumour. If regression was present on multiple tumour samples, the mean of the measurements made on all the slides was considered. In case of multiple regression foci on the same section, the sum of the different areas was considered. If regression was present in $<5 \%$, the lesion was considered as not regressed. Extensively regressed melanoma $(>75 \%)$ was not considered eligible for the study. Regression $>75 \%$ in horizontal extension according to the College of American Pathologists (CAP) definitions was not considered eligible for the study because in our experience it is present in only $4 \%$ of cases ${ }^{15}$ for this reason, we focused our analysis on the prognostic role of tumour regression $<75 \%$.

\section{Histological regression: distribution/topography}

In addition, the predominant distribution pattern of regression was evaluated (peripheral if present on the sides of the tumour, basal if distributed along the stromal-tumour interface, and intra-tumoural if central and completely surrounded by the neoplastic cells). In order to avoid an overlap with TIL, the key feature of regression was the evidence of destruction of neoplastic cells defined as 'necrosis or apoptosis or whole disappearance of malignant tumour cells or apparent partial or total active destruction of melanocytic tears with replacement by inflammatory cells or fibrosis or melanophages'. Areas of ulceration or those intimately associated or adjacent to it were not considered.

\section{Statistical analysis}

The specific inflammatory infiltrates (TIL-IRA, TIL-ORA, TIL-CTRL) features and associations with clinicopathological characteristics were assessed using the chi-square test and the non-parametric test. Specifically, the $p$ values in Table 1 were calculated as follows: if the variable had categorical data, the chi-squared test was performed; if the variable had continuous data, the Kruskal-Wallis rank sum test for median and the analysis of variance (ANOVA) for mean was performed. For more than two groups Tukey honestly significant difference (HSD) post-hoc test was performed. Logistic regression and post-estimation receiver operating characteristic (ROC) curve were used to define optimal cut-off of regression related to relapse $(>10 \%$, $>20 \%,>30 \%,>40 \%,>50 \%,>60 \%$ values were tested). Disease free interval (DFI) was calculated from the date of primary lesion diagnosis to the date of tumour progression/recurrence or last follow-up. Disease specific survival (DSS) was calculated from the date of primary lesion diagnosis to the date of death or last follow-up. Survival curves were estimated with the Kaplan-Meier method and compared using the log-rank test.

\section{RESULTS}

\section{Clinicopathological characteristics of enrolled patients}

Clinicopathological characteristics are reported in Table 1. Of the 70 patients included with histological regressed melanoma, $39(56 \%)$ were males and 31 (44\%) females. Median age was 58 years (range 23-78). Primary tumours were on the trunk in $58.6 \%$ of cases $(41 / 70)$, followed by lower limbs $(22 / 70,31.4 \%)$, upper limbs $(4 / 70,5.7 \%)$ and head/neck (3/70, 4.3\%). The most represented histotype was superficial spreading melanoma $(56 / 70,80 \%)$. Mean Breslow thickness was $2.73 \pm 2.10 \mathrm{~mm}$. The most represented Clark level was IV $(71.4 \%, 50 / 70)$. Ulceration was present in $33 \%$ of the lesions $(23 / 70)$. Mitotic count $\geq 1$ / $\mathrm{mm}^{2}$ was present in $61 \%(43 / 70) ; 38.5 \%(27 / 70)$ showed diffuse neo-angiogenesis and 48.6\% (34/70) had vascular invasion. In 20 cases $(28.6 \%)$, TILs were described as 'brisk'. There was metastatic involvement of the sentinel lymph node (SLN) in 37\% (26/70).

According to the AJCC classification, there were eight stage I (26\%), 26 stage II (37\%), 26 stage III (37\%) patients. After a median follow up of 4.9 years (range 0.7-8.9), 21/70 patients showed disease progression (30\%), and subsequently 15 died of melanoma during follow up $(21.4 \%)$. The pattern of first progression was: 12 regional metastases (12\%) [four lymph node metastases in the same SLN biopsy negative basin (false negative SLN biopsy), seven regional skin metastases, one lymph node metastasis into the parotid gland] and $13(18 \%)$ distant metastasis. During follow-up, of 12 patients with first progression at regional site, four were free of disease after the surgical resection of the metastasis, three patients showed a de novo skin recurrence, one patient had lymph node metastasis and four patients progressed in a distant site ( 2 in the lung, 2 in the liver).

\section{Histological regression evaluation}

\section{Percentage/ratio}

Median percentage of regression was $15 \%$ (range 5-75) (Table 1). After logistic regression and post-estimation ROC analyses, a cut-off value of $10 \%$ was identified as statistically significant towards a more favourable course [odds ratio (OR) 0.35 , confidence interval (CI) $0.12-0.98, p=0.048$ ]. Patients with less than $10 \%$ of regression showed a higher Breslow thickness compared to the others $(3.64 \pm 2.32 \mathrm{~mm}$ vs $2.29 \pm$ $1.85 \mathrm{~mm}, p=0.015)$, a higher prevalence of ulceration $(56 \%$ vs $21 \%, p=0.003$ ) and a higher prevalence of a mitotic count $\geq 1 / \mathrm{mm}^{2}$ ( $82 \%$ vs $51 \%, p=0.011$ ). There were no differences concerning other variables adopting the $10 \%$ cut-off value (Table 1). We identified 46 'early' and 24 'late' regressed 
Table 1 Clinical characteristics in regressed and not regressed melanomas

\begin{tabular}{|c|c|c|c|c|c|c|}
\hline & \multicolumn{3}{|c|}{ Histological regression stratified } & \multirow{2}{*}{$\begin{array}{l}\text { Histological regression } \\
\qquad \begin{array}{c}(5-75 \%) \\
(n=70)\end{array}\end{array}$} & \multirow{2}{*}{$\begin{array}{l}\text { No histological } \\
\text { regression (CTRL) } \\
(n=28)\end{array}$} & \multirow[t]{2}{*}{$p^{\mathrm{a}}$} \\
\hline & $5-10 \%$ & $10-75 \%$ & $p$ & & & \\
\hline Age, median (interval) & $55(34-78)$ & $59(23-78)$ & 0.551 & $58(23-78)$ & $53(27-83)$ & 0.432 \\
\hline Gender & & & 0.149 & & & 0.250 \\
\hline Female & 13 & 18 & & 31 & 16 & \\
\hline Male & 10 & 29 & & 39 & 12 & \\
\hline Primary site & & & 0.078 & & & 0.141 \\
\hline Head/neck & 2 & 1 & & 3 & 1 & \\
\hline Trunk & 9 & 32 & & 41 & 13 & \\
\hline Upper extremities & 1 & 3 & & 4 & 6 & \\
\hline Lower extremities & 11 & 11 & & 22 & 8 & \\
\hline Histotype & & & 0.245 & & & 0.291 \\
\hline SSM & 16 & 40 & & 56 & 23 & \\
\hline ALM & 5 & 6 & & 11 & 2 & \\
\hline Other & 2 & 1 & & 3 & 3 & \\
\hline Breslow thickness, $\mathrm{mm} \pm \mathrm{SD}$ & $3.64 \pm 2.32$ & $2.29 \pm 1.85$ & 0.015 & $2.73 \pm 2.10$ & $2.61 \pm 1.29$ & 0.769 \\
\hline $1-2$ & 5 & 14 & & 19 & 8 & \\
\hline $2-4$ & 5 & 14 & & 19 & 13 & \\
\hline$>4$ & 10 & 6 & & 16 & 4 & \\
\hline Clark & & & 0.688 & & & 0.586 \\
\hline 3 & 7 & 11 & & 18 & 10 & \\
\hline 4 & 15 & 35 & & 50 & 17 & \\
\hline 5 & 1 & 1 & & 2 & 1 & \\
\hline Ulceration & & & 0.003 & & & 0.351 \\
\hline No & 10 & 37 & & 47 & 16 & \\
\hline Yes & 13 & 10 & & 23 & 12 & \\
\hline Mitosis & & & 0.011 & & & 0.105 \\
\hline$<1$ & 4 & 23 & & 27 & 6 & \\
\hline$\geq 1$ & 19 & 24 & & 43 & 22 & \\
\hline Neoangiogenesis & & & 0.328 & & & 0.475 \\
\hline Absent/mild & 16 & 27 & & 43 & 15 & \\
\hline Present & 7 & 20 & & 27 & 13 & \\
\hline Inflammatory infiltrate & & & 0.013 & & & 0.795 \\
\hline Scarce & 14 & 14 & & 28 & 12 & \\
\hline Absent & 16 & 30 & & 46 & 14 & \\
\hline Present & 7 & 17 & & 24 & 14 & \\
\hline SLN status & & & 0.416 & & & 0.056 \\
\hline Negative & 16 & 28 & & 44 & 12 & \\
\hline Positive & 7 & 19 & & 26 & 16 & \\
\hline No. metastatic LN & & & 0.615 & & & 0.185 \\
\hline 0 & 16 & 28 & & 44 & 12 & \\
\hline $1-2$ & 6 & 16 & & 22 & 15 & \\
\hline $3-4$ & 0 & 2 & & 2 & 1 & \\
\hline$>4$ & 1 & 1 & & 2 & 0 & \\
\hline AJCC stage & & & 0.181 & & & 0.182 \\
\hline I & 4 & 14 & & 18 & 3 & \\
\hline II & 12 & 14 & & 26 & 10 & \\
\hline III & 7 & 19 & & 26 & 15 & \\
\hline Brisk infiltrate & & & 0.147 & & & 0.215 \\
\hline No & 19 & 31 & & 50 & 17 & \\
\hline Yes & 4 & 16 & & 20 & 12 & \\
\hline
\end{tabular}

AJCC, American Joint Committee on Cancer; ALM: acral lentiginous melanoma; LN, lymph node; SD, standard deviation; SLN, sentinel lymph node; SSM, superficial spreading melanoma.

${ }^{a}$ Refers to the total of regressed versus not regressed melanoma.

melanomas, respectively; no differences were observed on the basis of regression cut-off (17/23 early cases in $\leq 10 \%$ vs $29 / 47$ early cases in $10-75 \%, p=0.312$ ).

DFS was significantly longer in patients with more than $10 \%$ of histological regression: in particular, at 1 year of follow-up, $97.8 \%$ of the patients with $>10 \%$ regression were disease free compared to the $65.2 \%$ of patients with regression $<10 \%$, while at 5 years the rates were $73.1 \%$ and $48.7 \%$ $(p=0.005)$, respectively (Fig. 1A).

Concerning DSS, no significant difference was reached between regressed and not regressed tumours, even if the curve of the group with regression $>10 \%$ looked more favourable in terms of prognosis. Percentages of survival at 1 year were $97.8 \%$ vs $82.6 \%$ and $84.6 \%$ vs $68.2 \%$ at 5 years, respectively ( $p=0.07$ ) (Fig. $1 \mathrm{~B})$.

\section{TIL immunophenotype evaluation}

A total of 67 cases out of 70 histological regressed melanomas and 28 control melanomas were used for these analyses (Table 2), while three cases had insufficient material to perform all the determinations. 


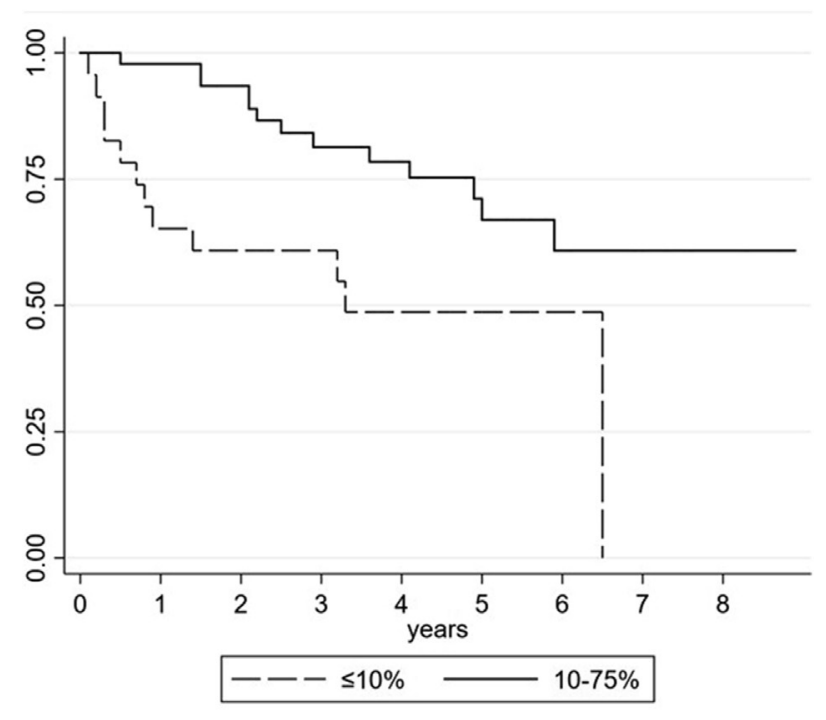

A)

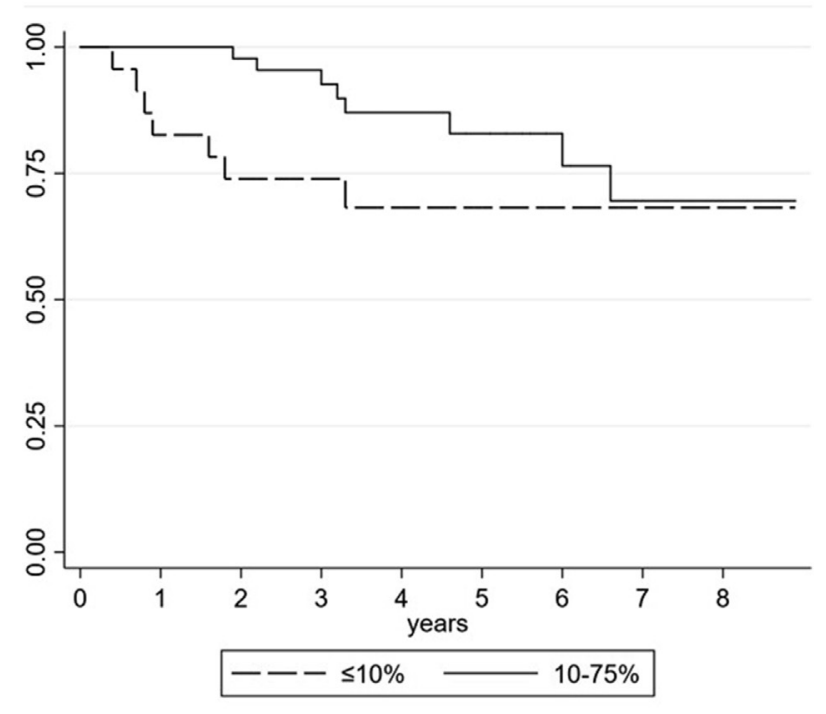

B)

Fig. 1 (A) Disease free interval and (B) disease specific survival of melanoma patients stratified for the extension of histological regression.

The control cohort of not regressed melanomas used for comparing the immunophenotype had a mean Breslow thickness of $2.61 \pm 1.29 \mathrm{~mm}$, the median age at diagnosis was 53 , and $12 / 28$ had ulceration of primary melanoma. There were no significant differences in the classical prognostic melanoma features between regressed and not regressed melanomas.

The distribution of immunophenotype in TILs in both regressed and not regressed lesions is reported in Table 2. No differences were observed in the immunophenotype between TIL-IRA and TIL-ORA in the 49 analysable cases when evaluating the CD3, CD4, CD8 sub-populations. Conversely, differences were detected in FOPX3/CD4 ratio in different subgroups $(10.1 \pm 9.28$ TIL-IRA vs $23.3 \pm 21.8$ TIL-ORA vs $38.4 \pm 25.4$ TIL-CTRL; $p<0.001$ ) (Fig. 2).

\section{FOXP3/CD4 evaluation and correlation with regression}

FOXP3/CD4 ratio was lower in TIL-IRA compared to TILORA $(10.1 \pm 9.28$ vs $23.3 \pm 21.8, p=0.0004)$, but both of them were significantly lower compared to TIL-CTRL (38.4 $\pm 25.4, p=0.0016)$ (Table 2). In particular, we evaluated the differences according to the extent of the regression $(\leq 10 \%$ vs $10-75 \%)$. FOXP3/CD4 ratio in TIL-CTRL (38.4 \pm 25.4$)$ was higher than the ratio observed in TILORA of $10-75 \%(21.8 \pm 17.9, p=0.0361)$ (Supplementary Table 1, Appendix A). When looking at differences of immunophenotype rate between TIL-CTRL and TIL-IRA, we observed a higher ratio of FOXP3/CD4 in the TILCTRL subset compared to both $\leq 10 \%$ and $10-75 \%$ categories $(38.4 \pm 25.4$ vs $9.7 \pm 9.87$ vs $10.3 \pm 9.10, p<0.001)$ (Supplementary Table 2, Appendix A). Supplementary Table 3 (Appendix A) reports data on regressed lesions: TIL-ORA ( $\leq 10 \%$ and $10-75 \%$ subgroups) showed a higher ratio of FOXP3/CD4 (26.0 \pm 28.2 and $21.8 \pm 17.9)$ compared to TIL-IRA ( $\leq 10 \%$ and $10-75 \%$ subgroups) $(9.7 \pm 9.87$ and $10.3 \pm 9.10)$

\section{PD1/CD4 evaluation}

PD1/CD4 ratio in TIL-IRA and TIL-ORA were significantly lower compared to TIL-CTRL $(4.9 \pm 5.5$ and $6.9 \pm 7.6$ vs $12.7 \pm 6.9, p<0.001$ ) (Table 2), while no difference was detected in PD1/CD4 ratio between TIL-IRA and TIL-ORA in regressed lesions $[4.9 \pm 5.5$ vs $6.9 \pm 7.6, p=$ not significant (ns)].

Similarly, PD1/CD4 ratio between TIL-IRA $\leq 10 \%$ vs TILIRA $10-75 \%$ was not statistically different $(5.7 \pm 5.3$ vs 4.5 $\pm 5.6, p=n s ;$ Supplementary Table 2, Appendix A). Moreover, PD1/CD4 ratio in TIL-ORA was higher in lesions with $\leq 10 \%$ of regression compared to TIL-ORA in lesions with $10-75 \%$ regression $(10.5 \pm 8.9$ vs $3.1 \pm 2.9, p=0.0004)$ (Supplementary Table 1, Appendix A).

When looking at differences in immunophenotype rates between TIL-CTRL and TIL-IRA in $\leq 10 \%$ or $10-75 \%$ categories, we observed a higher ratio of PD1/CD4 in TILCTRL compared to both $(12.7 \pm 6.9$ vs $5.7 \pm 5.3, p<0.007$; and $12.7 \pm 6.9$ vs $4.5 \pm 5.6, p<0.001$ ) (Supplementary Table 2, Appendix A). For TIL-CTRL and TIL-ORA in $\leq 10 \%$ or $10-75 \%$ categories, we observed a higher ratio of PD1/CD4 in TIL-CTRL compared to $10-75 \%$ regressed melanoma $(12.7 \pm 6.9$ vs $10.5 \pm 8.9, p=n s$; and $12.7 \pm 6.9$ vs $3.1 \pm 2.9$, p<0.0004) (Supplementary Table 1, Appendix A). In regressed lesions, (Supplementary Table 3, Appendix A) TIL-ORA in lesions with $\leq 10 \%$ showed a higher PD1/CD4 ratio compared to TIL-IRA in both categories.

\section{DISCUSSION}

Melanoma is considered an 'immunogenic' cancer because it is often associated with lymphocytic infiltration and has the ability of spontaneous regression. ${ }^{14-17}$ Those phenomena are strictly related, share some similarities, but probably represent different time-points and different mechanisms during the biological history of this tumour. Although the lymphocytic infiltrate has been described as a favourable prognostic feature, ${ }^{18}$ complete agreement on the role of histological regression has not been reached to date; indeed, either a negative or protective role on prognosis has been attributed to regression. ${ }^{4-6,19}$ Moreover, it has been 
Table 2 TIL immunophenotype in regressed and not regressed lesions

\begin{tabular}{|c|c|c|c|c|c|}
\hline Population & TIL-CTRL (A) $(n=28)$ & TIL-ORA (B) $(n=49)$ & TIL-IRA (C) $(n=67)$ & \multicolumn{2}{|c|}{ Tukey HSD post-hoc test } \\
\hline $\mathrm{CD} 3, \mathrm{n}^{\circ}$ cell $/ \mathrm{mm}^{2}$ & $523.8 \pm 386.4$ & $585.14 \pm 426.2$ & $500.2 \pm 337.3$ & 0.4874 & \\
\hline $\mathrm{CD} 4, \mathrm{n}^{\circ}$ cell $/ \mathrm{mm}^{2}$ & $238.3 \pm 159.9$ & $305.6 \pm 233.6$ & $278.0 \pm 182.5$ & 0.3570 & \\
\hline $\mathrm{CD} 8, \mathrm{n}^{\circ} \mathrm{cell} / \mathrm{mm}^{2}$ & $297.5 \pm 235.2$ & $299.9 \pm 224.6$ & $249.3 \pm 184.1$ & 0.8332 & \\
\hline $\mathrm{CD} 4 / \mathrm{CD} 8$ & $0.93 \pm 0.38$ & $1.11 \pm 0.61$ & $1.27 \pm 0.53$ & 0.0170 & A vs C 0.0148 \\
\hline $\mathrm{CD} 25, \mathrm{n}^{\circ} \mathrm{cell} / \mathrm{mm}^{2}$ & $38.9 \pm 37.6$ & $35.7 \pm 46.7$ & $27.1 \pm 27.2$ & 0.2696 & \\
\hline $\mathrm{FOXP} 3, \mathrm{n}^{\circ} \mathrm{cell} / \mathrm{mm}^{2}$ & $91.2 \pm 72.5$ & $62.8 \pm 73.9$ & $52.7 \pm 66.3$ & 0.0541 & A vs C 0.0420 \\
\hline $\mathrm{CD} 4 / \mathrm{CD} 3, \%$ & $49.8 \pm 17.2$ & $54.2 \pm 19.8$ & $58.8 \pm 18.3$ & 0.0872 & \\
\hline FOXP3/CD $4, \%$ & $38.4 \pm 25.4$ & $23.3 \pm 21.8$ & $10.1 \pm 9.28$ & $<0.001$ & $\begin{array}{l}\text { A vs B } 0.0016, \\
A \text { vs C }<0.001, \\
B \text { vs C } 0.0004\end{array}$ \\
\hline $\mathrm{PD} 1, \mathrm{n}^{\circ}$ cell $/ \mathrm{mm}^{2}$ & $28.4 \pm 42.5$ & $15.1 \pm 23.1$ & $12.9 \pm 21.1$ & 0.0380 & A vs C 0.0325 \\
\hline $\mathrm{PD} 1 / \mathrm{CD} 4, \%$ & $12.7 \pm 6.9$ & $6.9 \pm 7.6$ & $4.9 \pm 5.5$ & $<0.001$ & $\begin{array}{l}\text { A vs B } 0.0016, \\
A \text { vs } C<0.001\end{array}$ \\
\hline Melanophages, $\mathrm{n}^{\circ}$ cell $/ \mathrm{mm}^{2}$ & $77.2 \pm 81.8$ & $191.8 \pm 179.8$ & $191.8 \pm 179.8$ & 0.0017 & \\
\hline
\end{tabular}

HSD, honestly significant difference.

suggested that early immune activation could explain the different immunotherapy response rates in clinical practice. $^{20}$

After revision of slides and evaluation of the extent of regression, we identified a quantitative cut-off value (10\%) of histological regression associated with a different outcome. In fact, this cut-off maintained its prognostic role in terms of DFS by survival analysis, marking the difference between the not regressed $(\leq 10 \%)$ and the 'true' regressed melanomas
(10-75\%). A similar regression extent cut-off in dermoscopy has been associated with a different tumour molecular landscape in terms of copy number variation, which represents a marker of tumour aggressiveness. ${ }^{21}$ For DSS, no significant difference was reached between regressed and not regressed tumours; this finding can be related to overlaps with the widespread availability of immunomodulatory therapy in recent years, which might differentially affect survival in melanoma patients. ${ }^{22}$
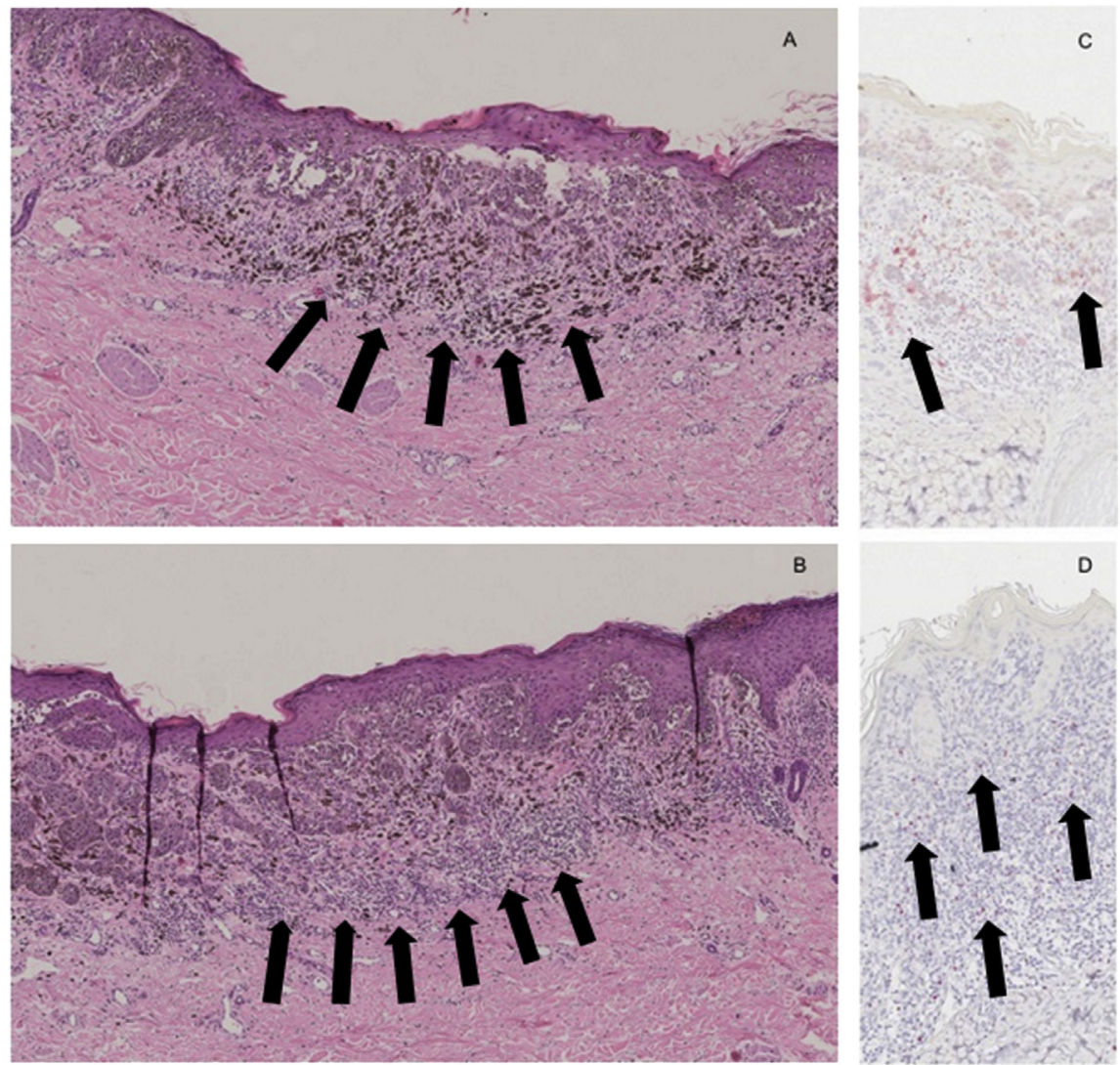

Fig. 2 (A) Low power H\&E of a regression area (IRA): a rich inflammatory infiltrate is present with lymphocytes and melanophages replacing neoplastic cells (arrows) (B) Low power H\&E of an area outside regression (ORA): an inflammatory infiltrate is present, although replacement of neoplastic cells is not (arrows). (C) Low power FOXP3 immunohistochemical staining showing few positive cells within the regression area (arrows). (D) Low power FOXP3 immunohistochemical staining showing multiple positive cells within the tumour infiltrating lymphocytes (arrows). 
In order to explain the different behaviour of regressed lesions, we analysed the immunophenotype of the recruited lymphocytes within and outside the 'regression' areas in regressed lesions compared to a control group without regression.

In our study, a lower rate of FOXP3/CD4 positive cells was observed in TIL-IRA and TIL-ORA in regressed melanoma compared to not regressed melanoma (TIL-CTRL). The FOXP3+ T-reg population has been described to decrease the innate and acquired response against the tumour through a mechanism which is physiologically involved in protecting against autoimmunity ${ }^{23,24}$ and has also been associated with different progression and survival rates in melanoma patients. ${ }^{18,24-26}$ Conversely, the absolute number of FOXP3+ cells was not significantly different between the groups in our series. When considering the FOXP3/CD4 ratio, it was inversely associated with histological regression, thus reflecting a different immunotolerance status between regressed and not regressed melanoma, potentially representing an early immunophenotypic marker of inactivation of the immune system. This finding is also supported by evidence that in metastatic melanoma FOXP3+ tumourinfiltrating immune cells are a potential predicting biomarker of response to treatment and survival. Balatoni et al. showed that the strongest predictor of ipilimumab treatment response was the density of FOXP3+ cells infiltrating lymph node metastases; moreover Di Gennaro et al. reported that electrochemotherapy induces a local response in skin melanoma metastasis by a lymphoid infiltrate characterised by a decrease in CD4+FOXP3+Treg cells. ${ }^{27-29}$

Similarly, we demonstrated a different count of PD1+ out of the total CD4+ count in the control group (without regression) compared to the regressed group. The PD1/PDL1 axis maintains a balance between tolerance and autoimmunity, thus cancer cells under selective pressure can exploit this signalling pathway with lymphocytes to decrease the immune response. PD1/PDL1 interaction inhibits $\mathrm{T}$ lymphocyte proliferation, survival, and effector functions, induces apoptosis of antigen-specific $\mathrm{T}$ cells, and promotes the differentiation of CD4+ T cells into FOXP3+ regulatory T cells. Moreover new evidence is suggesting that the peritumoural and the perimetastases infiltrate can be a potential therapeutic target in melanoma. ${ }^{26,30-33}$ From this point of view, the histological regression depends on the immune microenvironment and can be considered an indicator of a more efficient immune system exploitable for daily clinical practice. ${ }^{34}$

Limitations of the present study are due to the relatively low prevalence of regression in melanoma which, together with the need for adequate representative tissue, results in a relatively small sample size. Moreover, we considered only lesions with regression up to $75 \%$, because melanomas with regression higher than $75 \%$ are extremely rare and harbour uncertain clinical behaviour. ${ }^{15,35}$ The limited number of samples could help in explaining why statistical significance was not achieved in survival analysis, even though a trend was observed; this finding is probably also due to the increased use of immunomodulatory therapies in recent years. Another potential limitation could be the intrinsic operator-dependency in immunophenotype assessment, but we tried to overcome it by employing multiple independent observers.

Despite these potential drawbacks, based on these results we can suggest that histological regressed melanomas represent a heterogeneous group in terms of prognosis, possibly due to different immunomodulatory mechanisms which can affect the activity of TILs. Caution should be advised when interpreting the prognostic meaning of these characteristics, but an important observation is that a histological regression of $10-75 \%$ should be considered a biologically and clinically significant phenomenon impacting on survival. The observed heterogeneity in terms of immunophenotype between the different samples and tumour areas highlights the importance of target assessment and selection when devising future treatment strategies, and live adoptive cell therapy. ${ }^{36}$ Following our results, further studies are encouraged analysing the immunophenotype of histological regression, especially evaluating its correlation with response to therapies.

Conflicts of interest and sources of funding: Research at the Center of Translational Research in Melanoma at the University of Turin (Italy) is partially funded by Lanzavecchia-Lastretti Foundation and from Rete Oncologica Piemontese. This work was also supported by a grant from Ex60\% 2018 from from Italian Ministry for Education, University and Research (Ministero dell'Istruzione, dell'Università e della Ricerca, MIUR) to SOA and SR and received funding specifically dedicated to the Department of Medical Sciences from MIUR under the programme 'Dipartimenti di Eccellenza 2018-2022', Project no. D15D18000410001. The authors state that there are no conflicts of interest to disclose.

\section{APPENDIX A. SUPPLEMENTARY DATA}

Supplementary data to this article can be found online at https://doi.org/10.1016/j.pathol.2019.04.001.

Address for correspondence: Prof Paola Cassoni, University of Turin Medical Sciences Department, Section of Surgical Pathology, Via Santena 5 , Torino, 10126, Italy. E-mail: paola.cassoni@unito.it

\section{References}

1. Osella-Abate S, Ribero S, Sanlorenzo M, et al. Risk factors related to late metastases in 1.372 melanoma patients disease free more than 10 years. Int J Cancer 2014; 136: 2453-7.

2. Ribero S, Moscarella E, Ferrara G, et al. Regression in cutaneous melanoma: a comprehensive review from diagnosis to prognosis. $J$ Eur Acad Dermatol Venereol 2016; 30: 2030-7.

3. Socrier Y, Lauwers-Cances V, Lamant L, et al. Histological regression in primary melanoma: not a predictor of sentinel lymph node metastasis in a cohort of 397 patients. Br J Dermatol 2010; 162: $830-4$.

4. Ribero S, Osella-Abate S, Sanlorenzo M, et al. Favourable prognostic role of regression of primary melanoma in AJCC stage I-II patients. $\mathrm{Br} \mathrm{J}$ Dermatol 2013; 169: 1240-5.

5. Ribero S, Gualano MR, Osella-Abate S, et al. Association of histologic regression in primary melanoma with sentinel lymph node status: a systematic review and meta-analysis. JAMA Dermatol 2015; 151: $1301-7$.

6. Zugna D, Senetta R, Osella-Abate S, et al. Favourable prognostic role of histological regression in stage III positive sentinel lymph node melanoma patients. Br J Cancer 2018; 118: 398-404.

7. Requena C, Botella-Estrada R, Traves V, et al. Problems in defining melanoma regression and prognostic implication. Actas Dermosifiliogr 2009; 100: 759-66.

8. Danilova L, Wang H, Sunshine J, et al. Association of PD-1/PD-L axis expression with cytolytic activity, mutational load, and prognosis in melanoma and other solid tumors. Proc Natl Acad Sci USA 2016; 113: E7769-77.

9. Balch CM, Gershenwald JE, Soong SJ, et al. Final version of 2009 AJCC melanoma staging and classification. J Clin Oncol 2009; 27: 6199-206. 
10. Edge SB, Compton CC. The American Joint Committee on Cancer: the 7th edition of the AJCC cancer staging manual and the future of TNM. Ann Surg Oncol 2010; 17: 1471-4.

11. Rossi CR, Mozzillo N, Maurichi A, et al. Number of excised lymph nodes as a quality assurance measure for lymphadenectomy in melanoma. JAMA Surg 2014; 149: 700-6.

12. Massi G, LeBoit PE, editors. Histological Diagnosis of Nevi and Melanoma. 2nd ed. Heidelberg: Springer, 2014; 699-720.

13. Dessinioti C, Dimou N, Geller AC, et al. Distinct clinicopathological an prognostic features of thin nodular primary melanomas: an international study from 17 centers. J Natl Cancer Inst 2019; Mar 13: (Epub ahead of print).

14. Sznol M. Betting on immunotherapy for melanoma. Curr Oncol Rep 2009; 11: 397-404.

15. Ribero S, Galli F, Osella-Abate S, et al. Prognostic impact of regression in patients with primary cutaneous melanoma $>1 \mathrm{~mm}$ in thickness. $J A m$ Acad Dermatol 2019; 80: 99-105.

16. Gogas H, Ioannovich J, Dafni U, et al. Prognostic significance of autoimmunity during treatment of melanoma with interferon. $N$ Engl $J$ Med 2006; 354: 709-18.

17. Komenaka I, Hoerig H, Kaufman HL. Immunotherapy for melanoma. Clin Dermatol 2004; 22: 251-65.

18. Clemente CG, Mihm Jr MC, Bufalino R, et al. Prognostic value of tumor infiltrating lymphocytes in the vertical growth phase of primary cutaneous melanoma. Cancer 1996; 77: 1303-10.

19. Gualano MR, Osella-Abate S, Scaioli G, et al. Prognostic role of histological regression in primary cutaneous melanoma: a systematic review and meta-analysis. Br J Dermatol 2018; 178: 357-62.

20. Thumar JR, Kluger HM. Ipilimumab: a promising immunotherapy for melanoma. Oncology 2010; 24: 1280-8.

21. Gandolfi G, Longo C, Moscarella E, et al. The extent of whole-genome copy number alterations predicts aggressive features in primary melanomas. Pigment Cell Melanoma Res 2016; 29: 163-75.

22. Silva IP, Long GV. Systemic therapy in advanced melanoma: integrating targeted therapy and immunotherapy into clinical practice. Curr Opin Oncol 2017; 29: 484-92.

23. Franco-Molina MA, Miranda-Hernández DF, Mendoza-Gamboa E et al. Silencing of Foxp3 delays the growth of murine melanomas and modifies the tumor immunosuppressive environment. Onco Targets Ther 2016; 9: 243-53.

24. Quaglino P, Osella-Abate S, Marenco F, et al. FoxP3 expression on melanoma cells is related to early visceral spreading in melanoma patients treated by electrochemotherapy. Pigment Cell Melanoma Res 2011; 24: 734-6.

25. Mourmouras V, Fimiani M, Rubegni $\mathrm{P}$, et al. Evaluation of tumourinfiltrating $\mathrm{CD} 4+\mathrm{CD} 25+\mathrm{FOXP} 3+$ regulatory $\mathrm{T}$ cells in human cutaneous benign and atypical naevi, melanomas and melanoma metastases. Br J Dermatol 2007; 157: 531-9.

26. Weiss SA, Han SW, Lui K, et al. Immunologic heterogeneity of tumor infiltrating lymphocyte composition in primary melanoma. Hum Patho 2016; 57: 116-25.

27. Knol AC, Nguyen JM, Quéreux G, et al. Prognostic value of tumorinfiltrating Foxp3+ T-cell subpopulations in metastatic melanoma. Exp Dermatol 2011; 20: 430-4.

28. Balatoni T, Mohos A, Papp E, et al. Tumor-infiltrating immune cells as potential biomarkers predicting response to treatment and survival in patients with metastatic melanoma receiving ipilimumab therapy. Cancer Immunol Immunother 2018; 67: 141-51.

29. Di Gennaro P, Gerlini G, Urso C, et al. CD4+FOXP3+ T regulatory cells decrease and CD3+CD8+ T cells recruitment in TILs from melanoma metastases after electrochemotherapy. Clin Exp Metastasis 2016; 33: 787-98.

30. Topalian SL, Hodi FS, Brahmer JR, et al. Safety, activity, and immune correlates of anti- PD-1 antibody in cancer. N Engl J Med 2012; 366: $2443-54$.

31. Hodi FS, O'Day SJ, McDermott DF, et al. Improved survival with ipilimumab in patients with metastatic melanoma. N Engl J Med 2010 363: $711-23$

32. Robert C, Ribas A, Wolchok JD, et al. Anti- programmed-deathreceptor-1 treatment with pembrolizumab in ipilimumab-refractory advanced melanoma: a randomised dose-comparison cohort of a phase 1 trial. Lancet 2014; 384: 1109-17.

33. Ribero S, Longo C, Glass D, et al. What is new in melanoma genetics and treatment? Dermatology 2016; 232: 259-64.

34. Botella-Estrada R, Kutzner H. Study of the immunophenotype of the inflammatory cells in melanomas with regression and halo nevi. Am J Dermatopathol 2015; 37: 376-80.

35. Guitart J, Lowe L, Piepkorn M, et al. Histological characteristics of metastasizing thin melanomas: a case-control study of 43 cases. Arch Dermatol 2002; 138: 603-8.

36. Kelderman S, Heemskerk B, Fanchi L, et al. Antigen-specific TIL therapy for melanoma: a flexible platform for personalized cancer immunotherapy. Eur J Immunol 2016; 46: 1351-60. 